\title{
Identification and functional analysis of long non-coding RNAs in human and mouse early embryos based on single-cell transcriptome data
}

\author{
Jia-jun Qiu ${ }^{1}$, Zhao-rui Ren ${ }^{1,2}$, Jing-bin Yan ${ }^{1,2}$ \\ ${ }^{1}$ Shanghai Children's Hospital, Shanghai Institute of Medical Genetics, Shanghai Jiao Tong University School of Medicine, \\ Shanghai 200040, China \\ ${ }^{2}$ Key Laboratory of Embryo Molecular Biology, Ministry of Health of China and Shanghai Key Laboratory of Embryo and \\ Reproduction Engineering, Shanghai 200040, China
}

Correspondence to: Zhao-rui Ren, email: zhrren1225@163.com Jing-bin Yan, email: yanjingbin0130@hotmail.com

Keywords: long non-coding RNAs (IncRNAs), early embryo, WGCNA, single-cell RNA-seq, human embryogenesis

Received: January 21, $2016 \quad$ Accepted: August 08, 2016

Published: August 16, 2016

\section{ABSTRACT}

Epigenetics regulations have an important role in fertilization and proper embryonic development, and several human diseases are associated with epigenetic modification disorders, such as Rett syndrome, Beckwith-Wiedemann syndrome and Angelman syndrome. However, the dynamics and functions of long non-coding RNAs (IncRNAs), one type of epigenetic regulators, in human pre-implantation development have not yet been demonstrated. In this study, a comprehensive analysis of human and mouse early-stage embryonic IncRNAs was performed based on public single-cell RNA sequencing data. Expression profile analysis revealed that IncRNAs are expressed in a developmental stage-specific manner during human early-stage embryonic development, whereas a more temporal-specific expression pattern was identified in mouse embryos. Weighted gene co-expression network analysis suggested that IncRNAs involved in human early-stage embryonic development are associated with several important functions and processes, such as oocyte maturation, zygotic genome activation and mitochondrial functions. We also found that the network of IncRNAs involved in zygotic genome activation was highly preservative between human and mouse embryos, whereas in other stages no strong correlation between human and mouse embryo was observed. This study provides insight into the molecular mechanism underlying IncRNA involvement in human pre-implantation embryonic development.

\section{INTRODUCTION}

Understanding human pre-implantation development can not only provides insight into common human birth defects but also improve our understanding of the pathogenic mechanisms of many complex diseases such as Rett syndrome, Beckwith-Wiedemann syndrome and Angelman syndrome [1,2]. Thus, it is meaningful to understand the molecular mechanisms underlying preimplantation development.

Members of numerous non-coding RNA classes are expressed in the oocyte and pre-implantation embryo, and they have have an important role in fertilization and proper embryonic development [3], including directing cell fate decisions and cell differentiation during embryogenesis, which involves the formation of highly complex tissues comprised of many different cell types with specific and stable gene expression patterns [4]. Regulation of noncoding RNA occurs from the beginning of embryonic development. For example, the primary transcript of miR209 295, which is a miRNA cluster typically associated with the pluripotent state, is first detected in 4- to 8-cell embryos [5].

Long non-coding RNAs (lncRNAs), which are typically over 200 nucleotides in length, are involved in the cleavage stage of embryonic development [6]. Xist, the first identified lncRNA, is sufficient to trigger cis-inactivation of the $\mathrm{X}$ chromosome during the 4-cell stage, and another lncRNA; Fendrr, mediates long-term epigenetic marks to define expression levels of its target 
genes in mammalian embryogenesis [6-8]. Notably, a large number of human developmental disorders are related to the abnormal expression of some lncRNAs, such as $D B E-T$ in facioscapulohumeral muscular dystrophy, SNORD115 and SNORD116 in Prader-Willi Syndrome and KCNQ1OT1 and H19 in Beckwith-Wiedemann Syndrome and Silver-Russell Syndrome, which suggests that lncRNAs may play an important role in pre-implantation development [9-11]. However, the expression profiles and the regulation mechanism of lncRNAs in human earlystage embryos remain unclear.

Considering the limited availability of human oocytes and pre-implantation stage embryos, most studies of the functions of lncRNAs involved in embryonic development are based on model animals [12, 13]. A study of zebrafish embryogenesis found that a number of lncRNAs are involved in specific pathways and functions, ranging from cell cycle regulation to morphogenesis [12]. However, substantial differences in gene expression patterns exist between humans and model animals, which may limit the extrapolation of some findings to human embryonic development, especially for lncRNAs for which the sequence conservation is very low $[1,14]$. Nevertheless, the differences between lncRNA expression patterns and functions between human and model animals have not yet been clearly elucidated.

In this study, we elucidate the expression profiles and functions of lncRNAs in human early-stage embryos based on single-cell RNA sequencing (RNA-seq) data. We also compare the lncRNA expression profiles of human and mouse early-stage embryos. Genome-wide analysis of the functions of lncRNAs in pre-implantation stage will improve our understandings on the molecular mechanisms of human embryogenesis and developmental disorders.

\section{RESULTS}

\section{Transcriptome reconstruction from the single-cell RNA-seq data}

All reads of the 90 single-cell RNA-seq datasets (GSE36552) were aligned to the human genome (hg19) using HISAT, and the details of the mapping results are shown in Supplementary Table S1. The mapped reads were assembled into transcripts with the ab initio assembly software Cufflinks and Scripture (Figure 1, Supplementary Table S1). Mouse single-cell RNA-seq data (GSE44183) were aligned to the mouse genome (mm9) (Supplementary Table S1).

Subsequently, low-quality transcripts were removed using a read coverage threshold (see Materials and Methods). The corresponding receiver operating characteristic (ROC) curves are shown in Supplementary Figure S1. High-confidence transcripts were retained for downstream analysis.

\section{Identification of novel IncRNAs}

A novel lncRNA detection pipeline was developed to identify novel IncRNAs from the high-confidence transcripts. First, there were 94,418 and 77,464 unannotated transcripts were assembled by Cufflinks and Scripture, respectively. Among them, 535 transcripts which were assembled by both Cufflinks and Scripture were retained for downstream analysis. After size and exon number selection, 452 transcripts were selected as the putative novel lncRNAs. Finally, 421 transcripts were identified as novel lncRNAs based on their low coding potential as calculated with four different prediction tools (Figure 1 and Supplementary Dataset S1). These novel lncRNAs are listed in Supplementary Dataset S1.

\section{Transcriptional profiles across different stages}

We found that 15,400 and 6063 genes showed stagespecific expression (differential expression between any two consecutive stages) in the human and mouse datasets, respectively. There were notable differences in the human gene expression profiles between the 4- and 8-cell stages (Figure 2), which was consistent with the major maternalzygotic transition [15, 16]. Accordingly, significant differences were identified between the expression profiles of mouse pronuclei and the 2-cell stage (Figure S2). Two other dramatic changes were also authenticated in human transcript profiles. One was between the oocyte and zygote stages and the other was between the morula to late blastocyst at hatching stages (Figure 2A), which were likely caused by fertilization and cell differentiation, respectively.

\section{A more temporal-specific expression pattern was identified in IncRNAs rather than in protein-coding genes}

Previous studies have shown that lncRNAs are expressed in a tissue type-specific manner and that their expression levels are significantly lower than those of protein-coding genes [17]. In this study, the Spearman's rank correlation coefficients derived from IncRNA expression data were significantly lower than those of protein-coding genes, both in human and mouse embryos $\left(P\right.$-value $=2.2 \times 10^{-16}$, two-tailed Mann-WhitleyWilcoxon test; Figure 3A, Supplementary Dataset S2 and Supplementary Dataset S3), indicating that expression of IncRNAs was more variable than that of protein-coding genes in human early-stage embryonic development. Our analysis showed that the distributions of maximal JS (Jensen-Shannon, temporal specificity) scores for lncRNAs and protein-coding genes were significantly different, and IncRNAs were expressed in a more temporalspecific manner $\left(P\right.$-value $<2.2 \times 10^{-16}$, KolmogorovSmirnov test; Figure 3B, Supplementary Dataset S4 and 
Supplementary Dataset S5) in both human and mouse embryos. Using JS score $=0.5$ as a cutoff value, we found that $45.4 \%$ of IncRNAs were temporal-specific, relative to only $19.2 \%$ of protein-coding genes in humans ( $P$-value $<$ 0.001 , Fisher exact test). Our data also showed that temporal specificity scores for lncRNAs in mouse were significantly higher than those in human $\left(P\right.$-value $<2.2 \times 10^{-16}$, Kolmogorov-Smirnov test; Figure 3B, Supplementary Dataset S4 and Supplementary Dataset S5). However, the expression levels of lncRNAs were much lower than those of protein-coding genes $\left(P\right.$-value $<2.2 \times 10^{-16}$, KolmogorovSmirnov test; Figure 3C). Together, these observations suggest that lncRNAs exhibit more temporal specificity than protein-coding genes in human early embryos, and these differences were more pronounced in mouse early-stage embryonic development.

\section{IncRNAs may regulate gene transcription in cis in human embryo development}

Some lncRNAs may act in cis and affect the expression of genes in their chromosomal neighborhood [1820]. To test whether lncRNAs act in cis in human embryonic development, correlations between the expression patterns of lncRNAs and their neighbor coding genes, including 9440 unidirectional pairs and 3616 bidirectional pairs, were calculated. The results indicated a more correlation between lncRNAs and their coding neighbors than protein-coding gene-protein-coding gene pairs $\left(P\right.$-value $<2.2 \times 10^{-16}$, Kolmogorov-Smirnov test; $P$-value $<2.2 \times 10^{-16}$, Student's $t$-test, effect size $=0.61$; Figure $3 \mathrm{D}$ ). To confirm that this was a true cis effect of IncRNAs, we analyzed the correlations between the expression patterns of IncRNAs and their protein-coding gene neighbors and between protein-coding gene neighbors in two situations. The correlation between lncRNAs and protein-coding gene neighbors was significantly higher than between protein-coding genes and their proteincoding gene neighbors for both unidirectional neighbor-gene pairs $\left(P\right.$-value $<2.2 \times 10^{-16}$, Kolmogorov-Smirnov test; $P$-value $<2.2 \times 10^{-16}$, Student's $t$-test, effect size $=0.64$; mean correlation: 0.423 for $\operatorname{lncRNA}$-protein-coding gene pairs vs. mean correlation: 0.233 for protein-coding gene-proteincoding gene pairs; Supplementary Figure S3A) and divergent neighbor gene pairs $\left(P\right.$-value $<2.2 \times 10^{-16}$, KolmogorovSmirnov test; $P$-value $<2.2 \times 10^{-16}$, Student's $t$-test, effect size $=0.61$; mean correlation: 0.460 for lncRNA-protein-coding gene pairs vs. mean correlation: 0.251 for protein-coding gene-protein-coding gene pairs; Supplementary Figure S3B).

Taken together, these results confirmed that there were remarkably different expression patterns between lncRNA-protein-coding gene pairs and neighboring protein-coding gene pairs in both directions, which revealed that lncRNAs may regulate gene transcription in cis in human early-stage embryos.

\section{Functions of IncRNAs in human early-stage embryonic development}

To investigate the potential roles of lncRNAs in early-stage embryonic development, weighted gene co-expression network analysis (WGCNA) was performed on the stage-specific genes (Supplementary Dataset S6

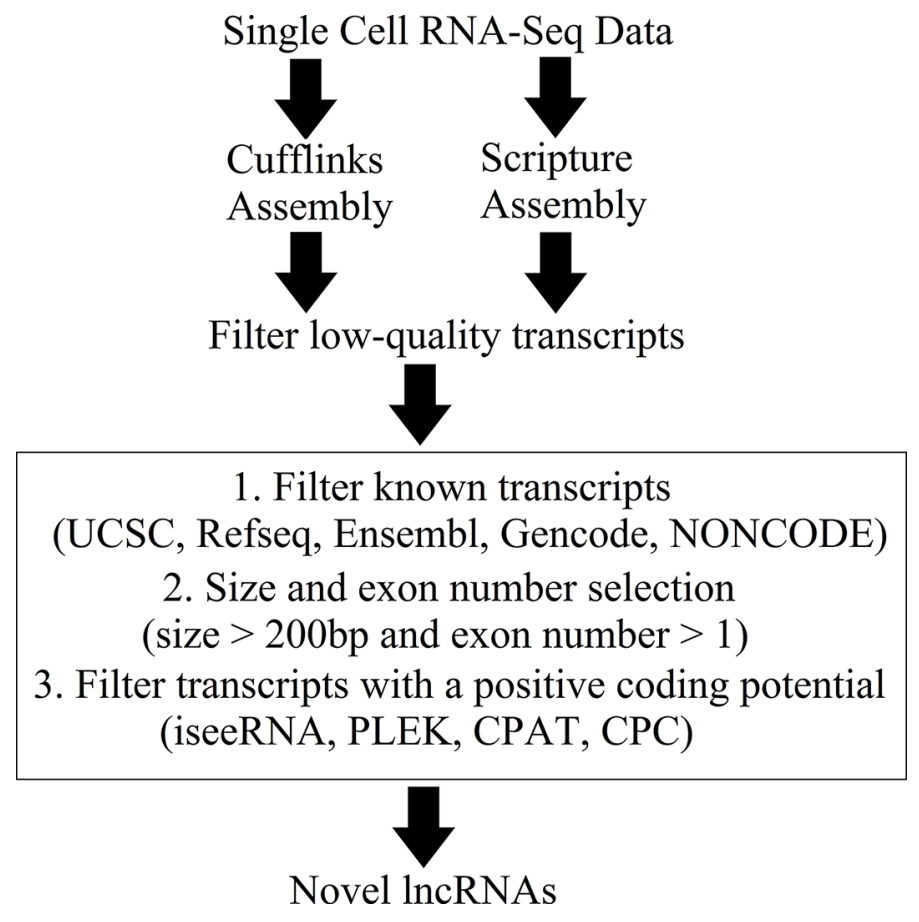

Figure 1: Overview of the novel IncRNA detection pipeline. 
and Supplementary Dataset S7). This analysis identified 17 modules in human embryos, 9 of which were highly correlated (correlation $>0.6, P$-value $<10^{-4}$ ) with specific developmental stages or with the entire developmental process (Figure 4, Figure S4 and Supplementary Dataset S8). In mouse embryos, six of eight modules were highly correlated with early embryonic development (Supplementary Figure S5, Supplementary Figure S6 and Supplementary Dataset S9). All of the modules contained a large number of lncRNAs (Figure 5A, Supplementary Dataset S8 and Supplementary Dataset S9). Enrichment analysis of GO terms and KEGG pathways within the modules was conducted (Figure 5B, Supplementary Dataset S10 and Supplementary Dataset S11). We also found that most of coding genes were the neighbor genes of lncRNAs in every modules ( $P$-value $=0.004$, Table 1), which validated the former result that lncRNAs may regulate gene transcription in cis in human early-stage embryos. Besides, we performed a hub-gene network analysis of each stage-specific module, and the interaction between hub lncRNAs and hub coding genes was also analyzed. The hub-lncRNAs were found in all stagespecific modules (Figure 6, Supplementary Dataset S8 and Supplementary Dataset S9), while some hub lncRNAs were found to co-localize with hub coding genes and cis-regulate them, and the others were confirmed to bind directly with the hub coding genes and trans-regulate them in almost all stage related modules (Figure 6, Supplementary Dataset S12 and Supplementary Dataset S13). The functions of these lncRNAs can be predicted based on the hub genes of known biological functions with which they were co-expressed with or bound.

\section{IncRNAs regulate human oocyte maturation}

One human module that contained a large number of lncRNAs (greenyellow; 464 lncRNAs and 759 protein- coding genes; Figure 5A) was highly correlated with the oocyte stage (Figure 4B). Genes in this module were enriched in the oocyte meiosis pathway $(\mathrm{KEGG}, P$-value $=$ 0.005, Supplementary Dataset S10). There were many hub coding genes which were related to oocyte maturation coexpressed with hub lncRNAs. Some of them were bound directly by hub lncRNAs, such as AURKA, BCL2L10, and the others such as TNFSF13, were the neighbor genes of hub lncRNAs [21-23] (Figure 6, Supplementary Dataset S8, Supplementary Dataset S12). Thus, hub lncRNAs of this module may be important for the regulation of oocyte maturation. Notably, lncRNAs in this module are related to protein ubiquitination (GO enrichment, $P$-value $=0.001$; Figure 5B and Supplementary Dataset S10) and regulation of protein ubiquitination (GO enrichment, $P$-value $=0.02$; Figure 5B and Supplementary Dataset S10), which agrees with previous reports that the ubiquitin-proteasome pathway (UPP) can control oocyte meiotic maturation $[24,25]$. These results suggested that lncRNAs may activate oocyte maturation and meiosis.

\section{IncRNAs involved in human zygotic genome activation}

Zygotic genome activation (ZGA) occurs between the 4- and 8-cell stages of human embryonic development and is the point at which zygotic transcripts gradually take control of development as maternal transcripts are degraded $[1,26]$. In our study, half of human stage-specific modules were related to the 4- and 8-cell stages, and these modules contained a large fraction of lncRNAs. Genes in the human modules highly correlated with the 4-cell stage (pink) were enriched in GTPase, which mediates signal transduction (Figure 5B and Supplementary Dataset S10). There were many hub coding genes which were related to GTPase
A

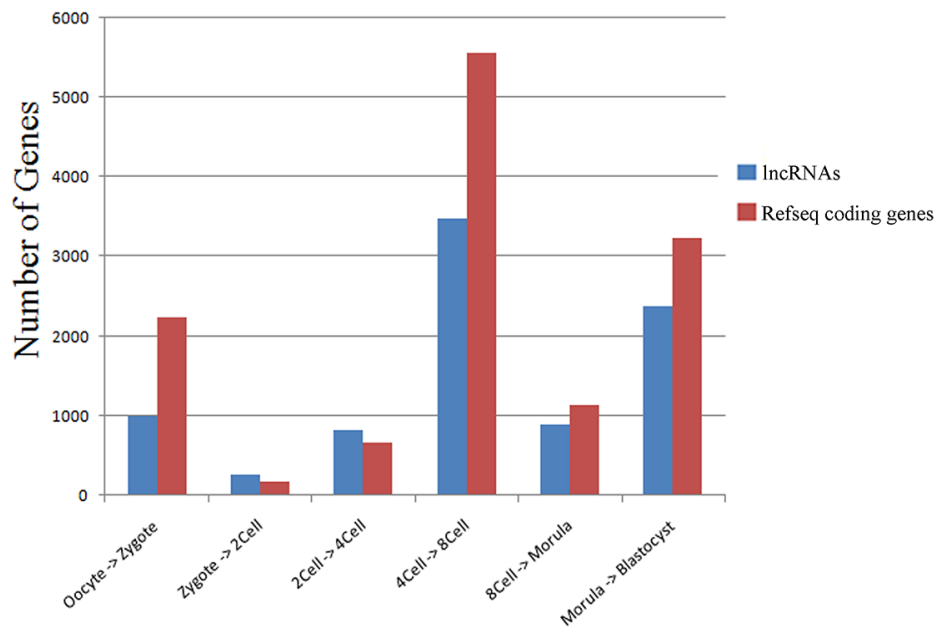

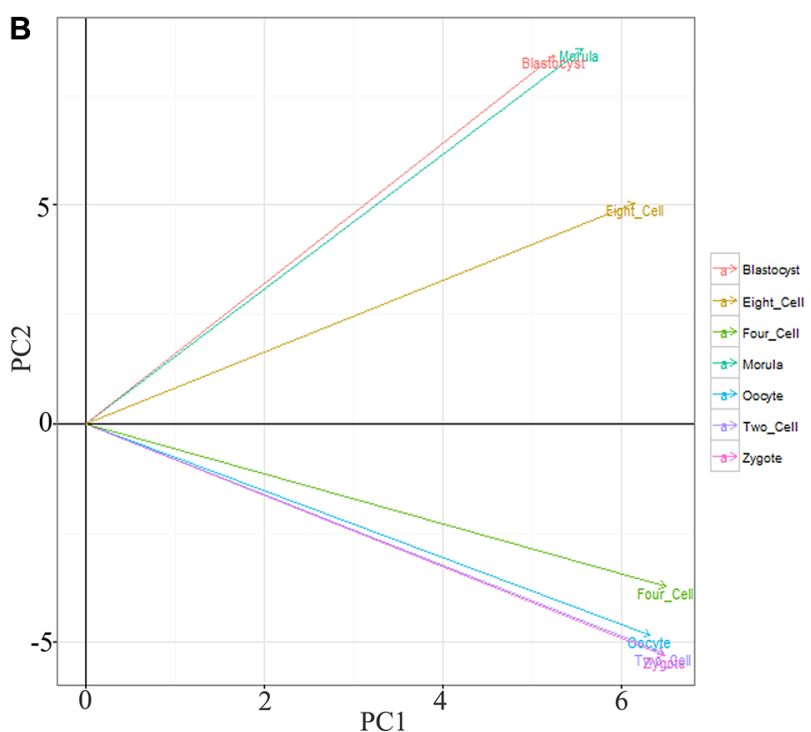

Figure 2: Global gene expression patterns during the seven consecutive stages of human pre-implantation development. (A) Bar graph showing the total number of differentially expressed genes between successive developmental stages $(q$-value $<0.01$ and $\log _{2}$ fold change $>1$ ). (B) Principal component (PC) analysis based on lncRNA expression profiles of human pre-implantation embryos. 
co-expressed with hub lncRNAs. Some of them were bound directly by hub lncRNAs, such as RASA3, and the others such as $G N G 2$, were the neighbor genes of hub lncRNAs (Figure 6, Supplementary Dataset S8, Supplementary Dataset S12). The human modules related to the 8-cell stage (magenta, brown and yellow) were enriched in several functions related to ZGA, including nucleosome assembly (GO enrichment, $P$-value $=8.19 \times 10^{-11}$; Figure 5B and Supplementary Dataset $\mathrm{S} 10)$ and chromatin assembly (GO enrichment, $P$-value $=$ $5.05 \times 10^{-10}$; Figure 5B and Supplementary Dataset S10). In conclusion, we found that some hub lncRNAs may activate ZGA during the human 4- and 8-cell stages.

\section{IncRNAs regulate mitochondrial functions}

Basic research in model species and clinical in vitro fertilization studies have shown that mitochondria play an important role in the regulation of mammalian early embryogenesis and that embryonic mitochondrial
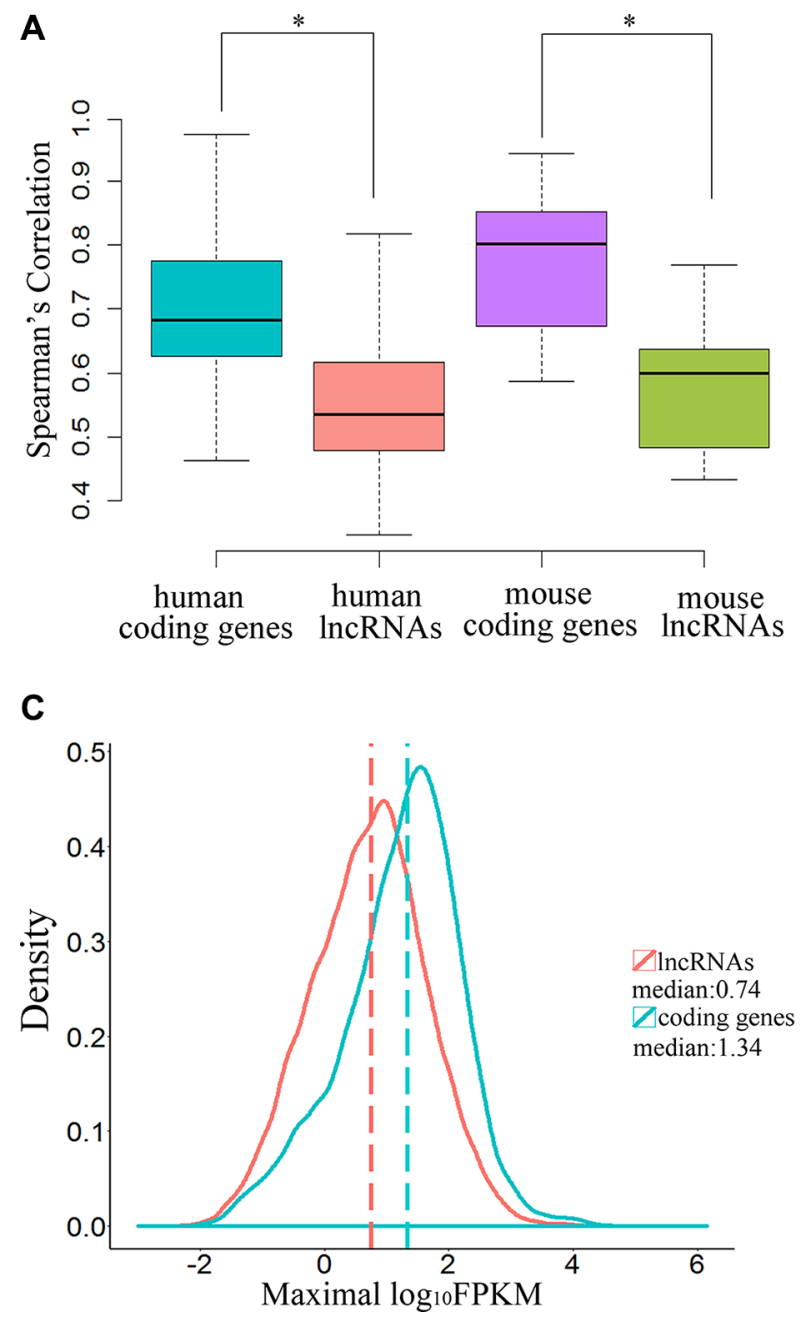

replication occurs after the hatched-blastocyst stage $[27,28]$. In this study, we found that the gene functions in two human blastocyst stage-related modules (black and green) were both enriched in mitochondrion functions (Figure 5B and Supplementary Dataset S10). Hub lncRNAs in these two modules bound directly and coexpressed with several mitochondrial function genes, including ATP5G3, COX4I1 and NDUFS6, which indicated that hub lncRNAs of blastocyst modules may correlate to mitochondrial function (Figure 6, Supplementary Dataset S8, Supplementary Dataset S12) [29].

\section{Comparison of IncRNA functions between human and mouse early-stage embryonic development}

Because we found that lncRNAs were expressed in a more temporal-specific manner in mouse early embryos than that in human embryos, we also analyzed

$\mathbf{B}$
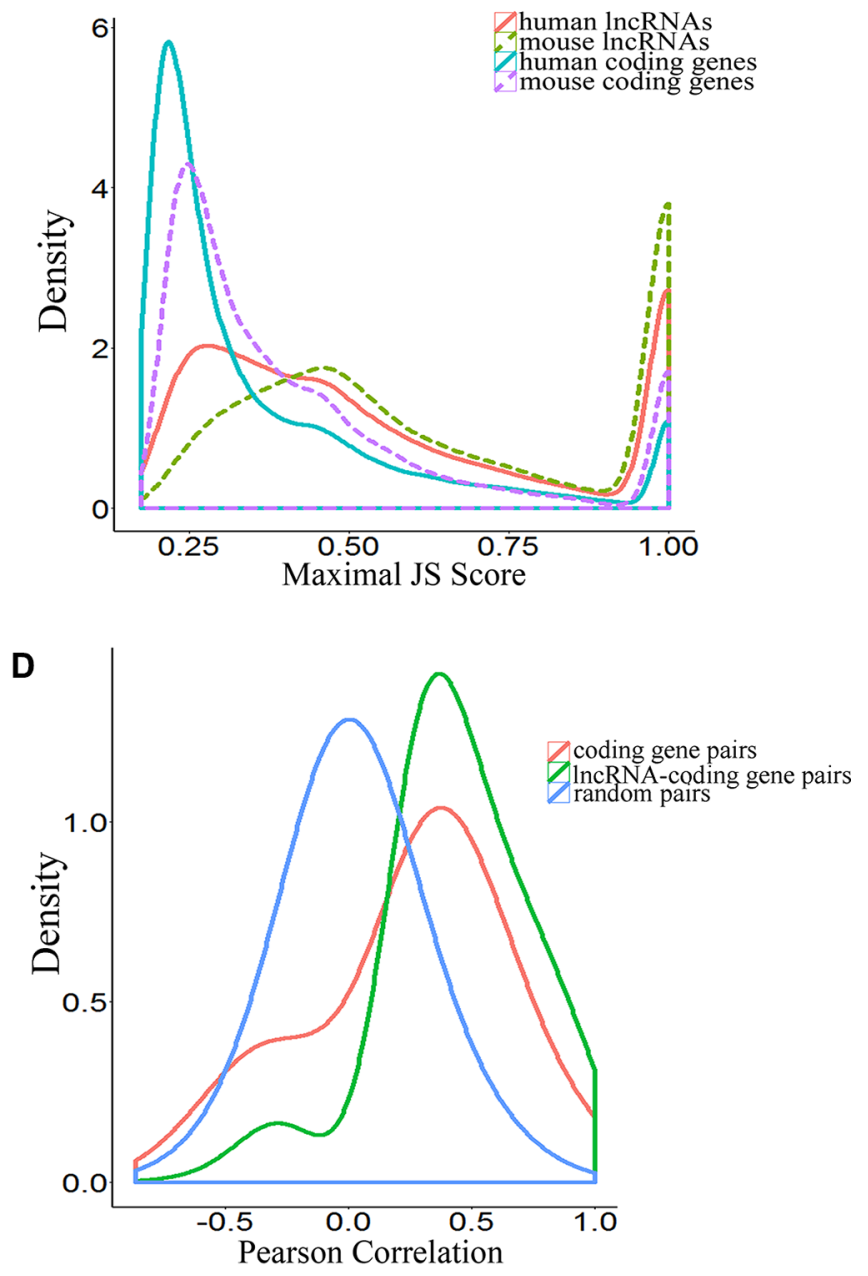

Figure 3: Temporal-specific expression of IncRNAs. (A) Boxplot indicating the distribution of Spearman's rank correlation coefficients between each embryonic sample pair derived from lncRNAs and coding genes (* means $P$-value $<0.05$ ). (B) Distribution of JSDbased specificity of genes in various stages. (C) Distribution of maximal expression $\left(\log _{10}\right.$-normalized FPKM counts estimated by Cufflinks) of lncRNAs and coding genes in human pre-implantation development. (D) Pearson correlation coefficient distributions for expression levels across the samples in human pre-implantation development. The random pairs are 10,000 random pairs of protein-coding genes. 
the functions of lncRNAs in mouse early embryonic development. Though lncRNAs were also found to be related to several functions in specific stages during mouse early embryonic development, the functions of lncRNAs were not identical in mouse or human embryonic development. For example, lncRNAs in the human 8-cell stage appear to have an important role in GTPase mediated signal transduction (Figure 5B and Supplementary Dataset S10), whereas lncRNAs in the mouse 8-cell stage do not appear to have such functions (Supplementary Dataset S11). Comparing the human modules to the mouse developmental data, we found that the human 8 -cell modules overlapped significantly with the mouse 2-cell module, during which mouse ZGA occurs (Figure 7). While mouse pre-major ZGA genes are spread over the longer gestational pre-major ZGA stage in humans. Likewise, post-major ZGA networks are found to have significant overlap and spread throughout all post-major ZGA human stages (Figure 7). These results suggested that the networks (modules) of lncRNAs involved in ZGA are particularly conserved between human and mouse embryos, but there is less preservation across other stages.

\section{DISCUSSION}

A number of diseases are caused by the disruption of epigenetic regulation during early embryonic development $[3,30]$. However, no systematic studies focused on the functions of lncRNAs during human early embryonic development has been described. Here we identified 421 novel lncRNAs in the first study to determine expression levels of all genes in human early-stage embryos.
Furthermore, we found that lncRNAs are expressed in a developmental stage-specific manner, and they may regulate gene transcription in cis in human embryonic development.

Oocyte meiosis is a specialized cell cycle that gives rise to fertilizable haploid gametes and is precisely controlled on many levels. Previous studies have found that the UPP regulates both human and mouse oocyte meiotic maturation in several ways [25, 31]. CRL4DCAF1 ubiquitin E3 ligase facilitates oocyte meiotic maturation by proteasomal degradation of the protein phosphatase 2A scaffold subunit PP2A-A, which inhibits cohesin removal and homologous chromosome separation during meiosis I [24]. UPP also has roles in oocyte meiotic maturation, because the degradation of cyclin B1 mediated by UPP is necessary for disjunction of pairs of homologous chromosomes during the first meiotic division in oocytes [25]. In hub-gene network analysis, we found 60 lncRNAs in the regulation network module built on co-expression with 10 UPP genes, among them 30 lncRNAs bound directly with the 9 UPP genes (Figure 6F). Thus lncRNAs may activate oocyte meiotic maturation through regulating the UPP in both human and mouse. With these results, we have a better understanding of the mechanisms of oocyte meiotic maturation and oocyte maturation failure [32].

The ZGA is another important early embryonic developmental period in which maternal mRNAs are cleared and embryonic transcription is activated [26]. Remodeling of chromatin surrounding nucleosomes, including repositioning of nucleosomes and post-translational modifications of histones, coincides with ZGA, which leads to exposure of the transcription start sites of zygotic genes
A

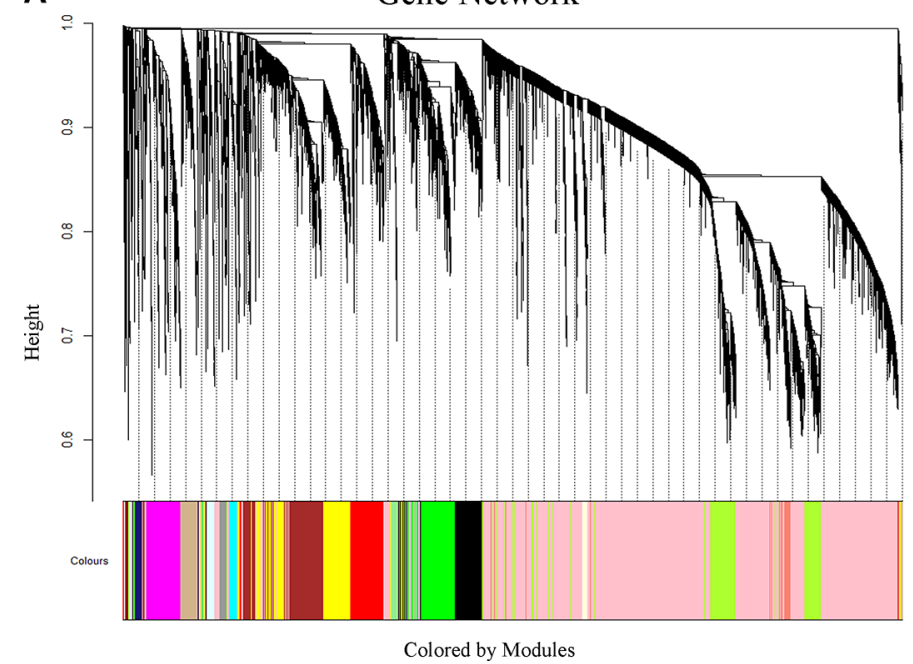

\section{B}

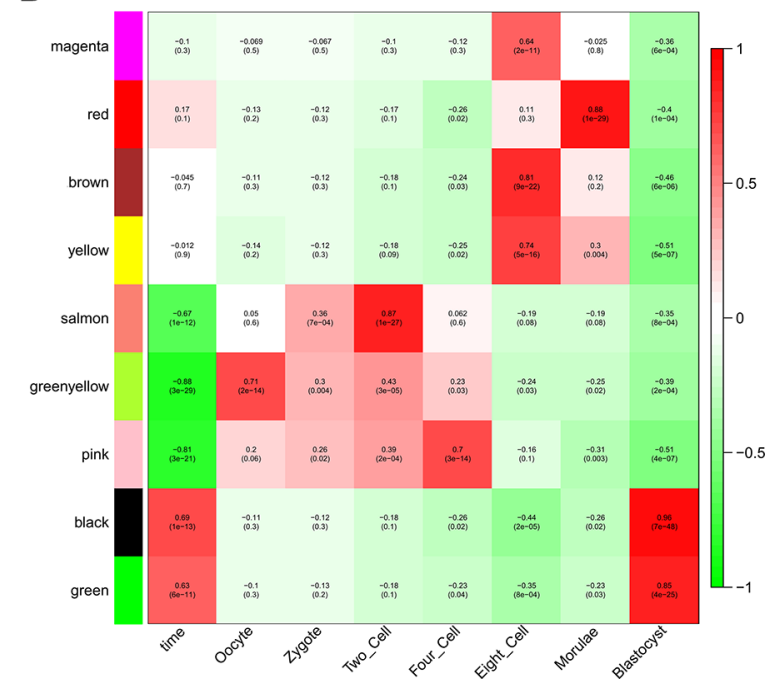

Figure 4: Network analysis of human pre-implantation development. (A) Hierarchical cluster tree showing co-expression modules identified using WGCNA. Modules correspond to branches and are labeled by colors as indicated by the color band underneath the tree. (B) Heatmap of correlations followed by the $P$-values in parentheses between modules and developmental stage. The color of each square corresponds to the degree of correlation: positive correlation, red; negative correlation, green; no correlation, white. The "time" column on the left represents the correlation of each module with the entire development process. 
and permits recruitment of the RNA polymerase II complex [26]. Previous studies have shown that lncRNAs can regulate chromatin remodeling and recruitment of the RNA polymerase II $[33,34]$. For example, lncRNA SChLAP1 antagonizes SNF5 (also known as SMARCB1), an essential subunit that facilitates SWI/SNF binding to histone protein
[35]. In this study, we confirmed that IncRNAs may stimulate ZGA through regulating nucleosome assembly and chromatin assembly during ZGA.

As the mouse is a wildly used animal model for human disease research, we compared human and mouse stage-specific modules in this study. We found

A

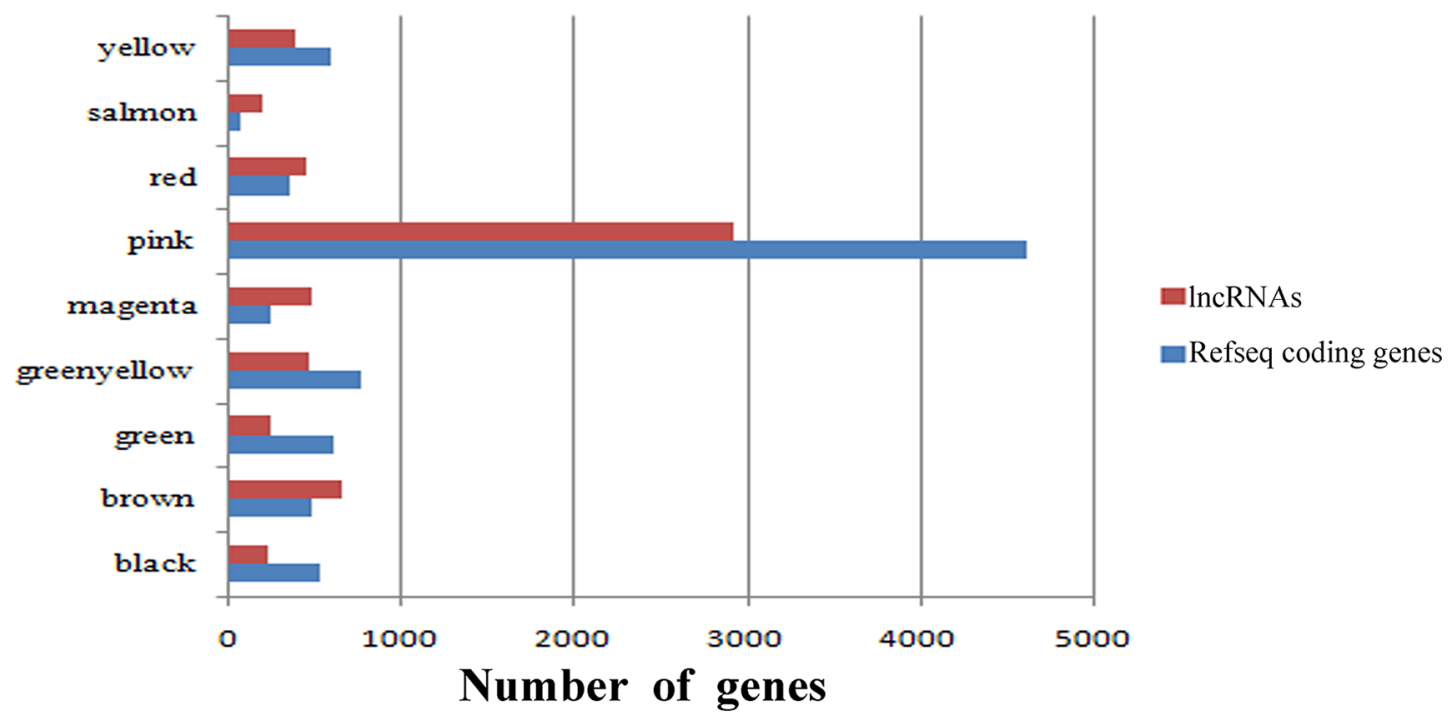

B

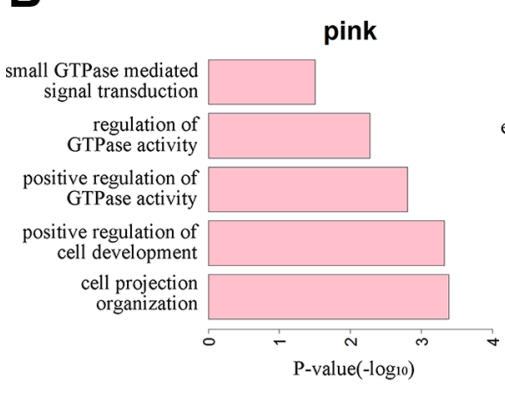

magenta

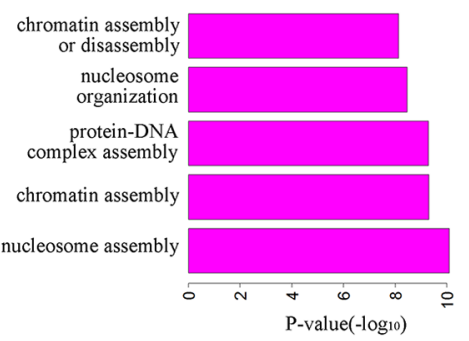

greenyellow

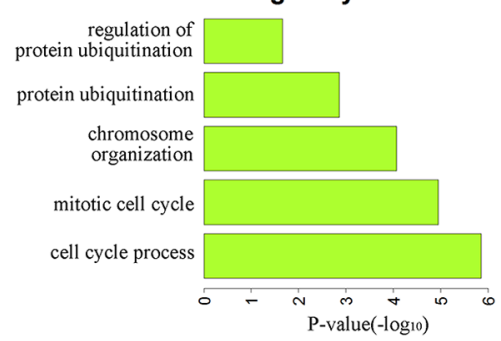

black

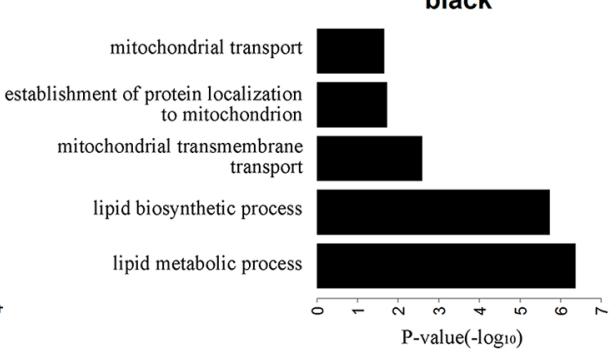

green

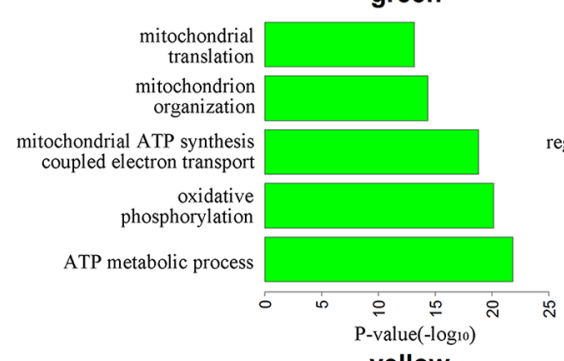

yellow

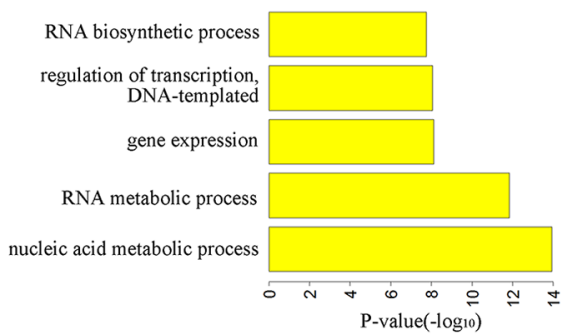

brown

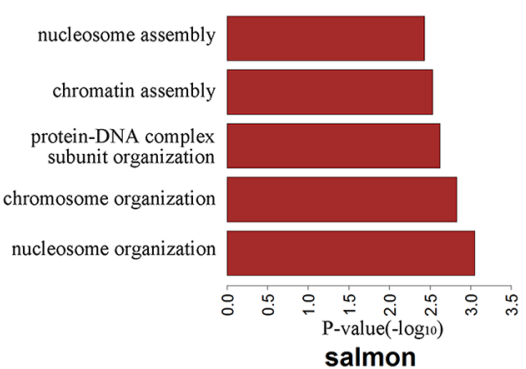

biological regulation

regulation of biosynthetic process regulation of cellular biosynthetic process

chromatin modification

histone modification

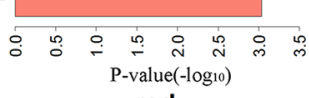

red

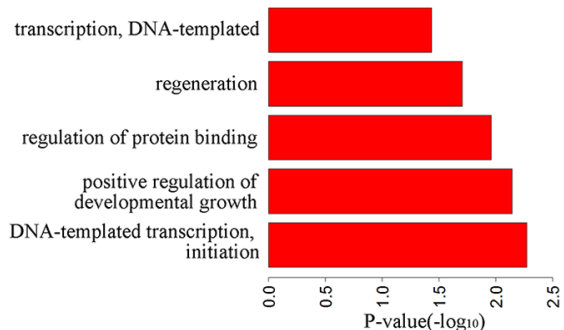

Figure 5: Function prediction of IncRNAs involved in pre-implantation development. (A) Bar graph showing the number of IncRNAs and coding genes in each module. (B) Bar plots showing GO enrichment in the modules. The length of the bars indicates the significance. 
Table 1: Coding genes were the neighbor genes of IncRNAs in every modules

\begin{tabular}{ccccc}
\hline Module & Neighbor coding genes (\%) & Non- neighbor coding genes (\%) & $\boldsymbol{t}$ & $\boldsymbol{P}$-value \\
\hline black & 56.23 & 43.77 & 3.970 & 0.004 \\
brown & 59.46 & 40.54 & \\
green & 54.13 & 45.87 & \\
greenyellow & 55.20 & 44.80 & \\
magenta & 52.81 & 47.19 & \\
pink & 63.21 & 36.79 & \\
red & 57.43 & 42.57 & \\
salmon & 47.37 & 52.63 & \\
yellow & 56.57 & 43.43 & \\
Mean & $55.82 \pm 4.4$ & $44.18 \pm 4.4$ & \\
\hline
\end{tabular}

A

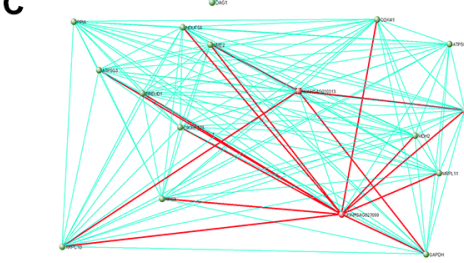

E

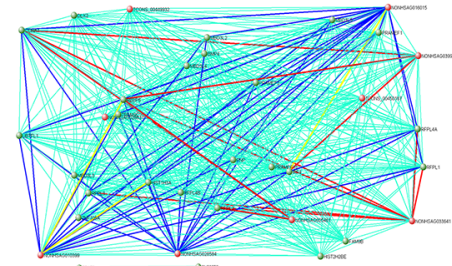

G
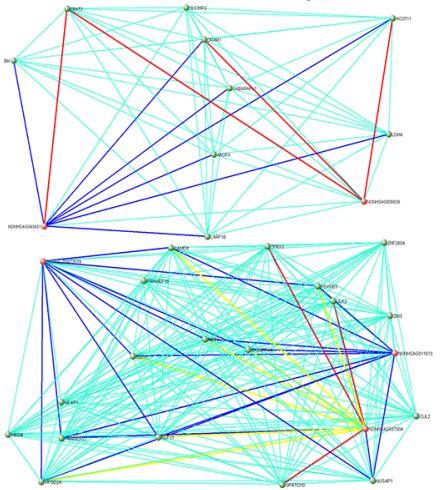

B

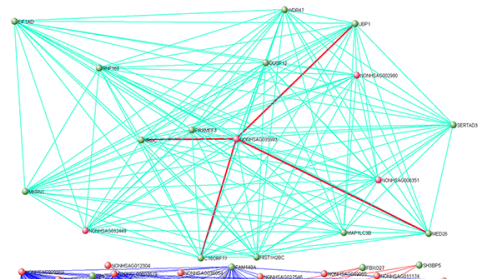

D

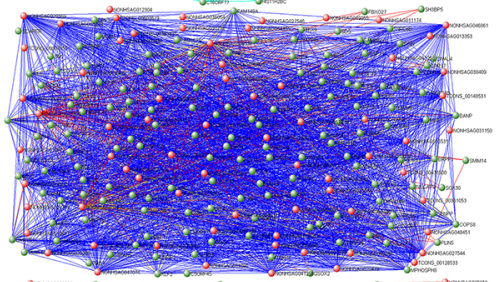

$\mathbf{F}$

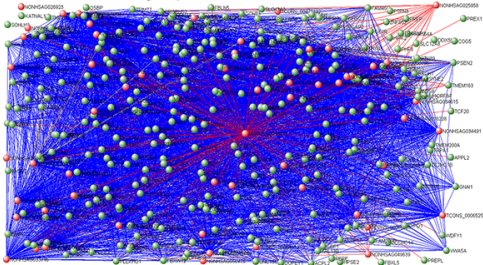

H

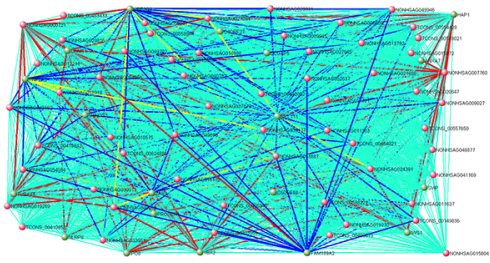

Figure 6: Hub gene networks for human stage-specific modules. Visualization of gene-gene interactions within each module. The connections were drawn using the VisANT tool. The genes with at least one connection when the weighted cutoff value was $\geq 0.1$ are shown. Each node represents a hub gene. The red nodes are hub lncRNAs. The green edges mean co-expression, and the red edges mean both co-expression and interaction, and the blue edges mean both co-expression and neighbor gene, and the yellow edges mean all three situation (co-expression, interaction and neighbor gene). To make the background clear, the green edges are not shown in Figure D and F. (A) Black module. (B) Brown module. (C) Green module. (D) Greenyellow module. (E) Magenta module. (F) Pink module. (G) Red module. (H) Salmon module. (I) Yellow module. 
that the networks of lncRNAs in the human 8-cell stage were particularly similar to those in the mouse 2-cell stage, and the pre- and post-ZGA modules in human and mouse overlapped across multiple stages. This probably reflects species-specific differences in human and mouse gestational periods and/or the very low sequence conservation of lncRNAs, because major differences in transcript structure result in functional differences [14]. Because of the large differences between human and mouse lncRNA networks, except those involved in ZGA, the value of research in mice may be limited, and it will be important to examine the functions of lncRNAs in human early embryonic development directly.

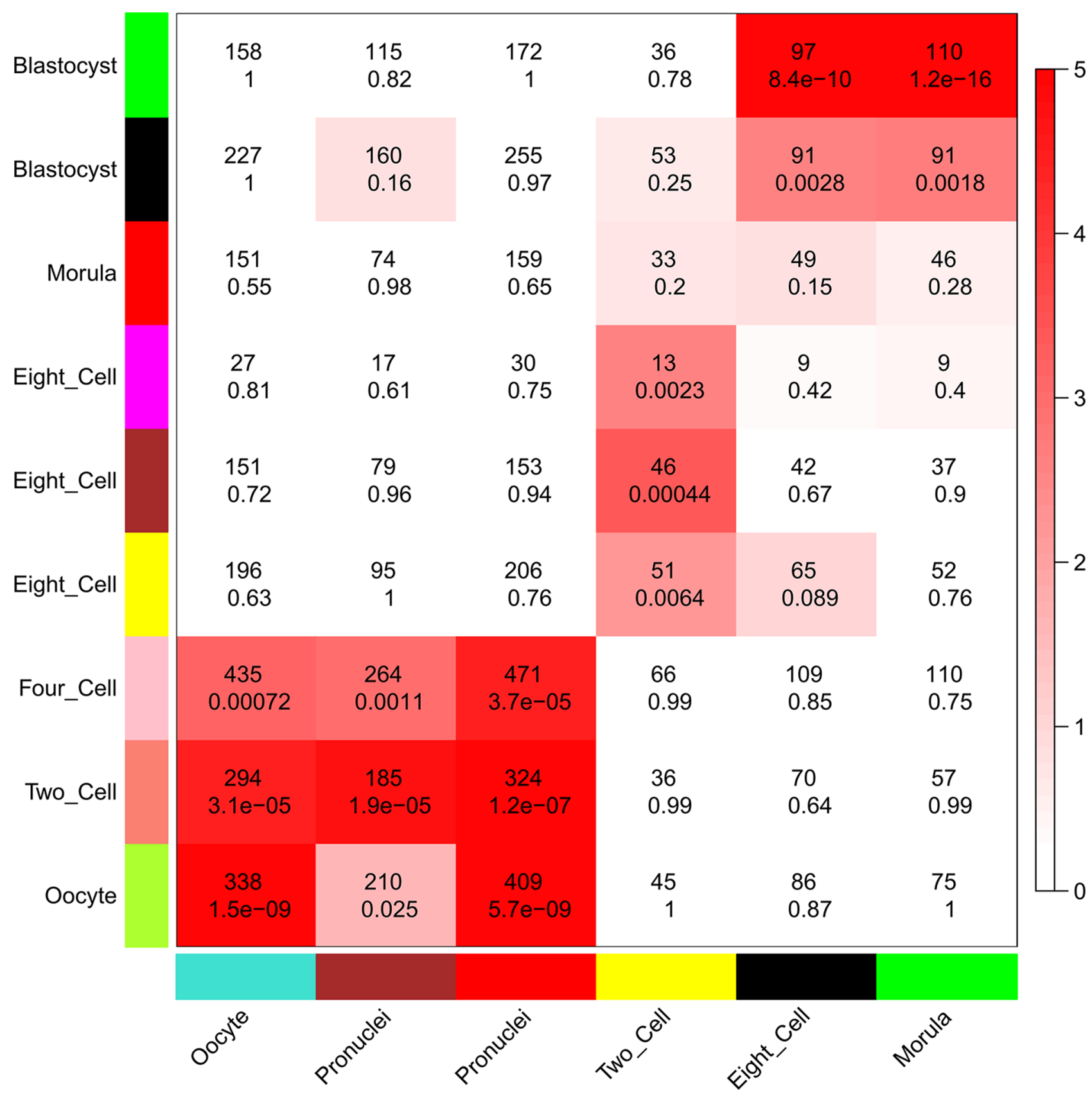

Figure 7: Comparison of modules in human and mouse early embryonic development. Heatmap showing the significance of gene overlaps between independently constructed human and mouse modules. The $x$ axis shows only mouse stage-specific modules $(n=6)$, and the $y$ axis shows all human modules $(n=9)$. Each cell contains the number of intersecting genes and the $P$-value of the intersection. Color legend represents $-\log _{10}$-transformed $P$-values based on a hypergeometric test. 


\section{MATERIALS AND METHODS}

\section{Single-cell RNA-seq dataset}

The single-cell RNA-seq dataset of human earlystage embryos (GSE36552) was downloaded from the Gene Expression Omnibus (GEO) of the National Center for Biotechnology Information. The dataset consists of 90 samples from seven crucial stages of embryo development: 3 metaphase II oocyte samples, 3 zygote samples, 6 2-cell stage samples, 12 4-cell stage samples, 20 8-cell stage samples, 16 morula stage samples and 30 late blastocyst at hatching stage samples [16]. The single-cell RNA-seq dataset of mouse early-stage embryos (GSE44183), which includes 17 samples ranging from the oocyte to morula stages, was also downloaded from the GEO. Both of these datasets were generated with the Illumina HiSeq 2000 system [36].

\section{Data pre-processing and filtering}

\section{Read mapping and transcript assembly}

Reads were aligned to the human (hg19) and mouse (mm9) genomes by HISAT (version 1.4.1; a successor to TopHat2), which is the fastest aligning algorithm currently available and one of the most accurate [37].

Aligned reads from HISAT were then assembled into transcripts separately by two different approaches: Cufflinks (version V2.2.1) and Scripture (beta version 2). Cufflinks uses a probabilistic model to simultaneously assemble and quantify the expression level of a minimal set of isoforms and provides a maximum likelihood explanation of the expression data in a given locus. Scripture uses a statistical segmentation model to distinguish expressed loci from experimental noise and uses spliced reads to assemble expressed segments. It reports all statistically significantly expressed isoforms in a given locus. The two approaches might generate different results in terms of assembled transcripts and numbers of products [38].

Cufflinks version V1.0.3 was run with default parameters (and 'min-frags-per-transfrag $=0$ ') and Scripture version 1.0 was run with default parameters [38-40].

\section{Filtering low-quality transcripts}

To remove low-quality reconstructed transcripts, transcripts assembled by Cufflinks with coverage below 4.03418 reads per base were eliminated (the threshold of transcripts assembled by Scripture was 1.39501). This minimal read coverage threshold was calculated by the method described previously [17, 41]. Transcripts that recovered $75 \%$ of annotation were regarded as good reconstructed transcripts. The ROC curve was used to evaluate the performance of different coverage thresholds between good and bad reconstructed transcripts. The final threshold was the average of the optimum threshold for coding ('NM' prefix) and non-coding ('NR' prefix) RNAs in RefSeq (NCBI Reference Sequence Database).

\section{Calculating optimum coverage threshold}

A coverage threshold set $T$ with specified sensitivity and specificity was generated with the $R$ package pROC based on the coverage values of good and bad reconstructed transcripts [42]. The index of the optimum coverage threshold in set $T$ can be obtained by formula 1 , in which $i^{*}$ represents the index of the optimum coverage threshold and sensitivities [i] and specificities [i] respectively denote the sensitivities and specificities of the $i$ th coverage threshold. The value for $i$ is enumerated in $I$, ranging from 1 to the size of the coverage threshold set $T$. Then the optimum coverage threshold can be obtained with formula 2 [43].

$$
\begin{aligned}
& i^{*}=\operatorname{argmin}_{i \in I}\left\{\sqrt{(1-\text { sensitivities }[i])^{2}+(1-\text { specificities }[i])^{2}}\right\} \\
& t^{*}=T\left[i^{*}\right]
\end{aligned}
$$

\section{Novel IncRNA detection pipeline}

The novel lncRNAs were obtained by the following steps: (1) Cuffcompare in Cufflinks was run using default parameters (and '-M discard (ignore) single-exon transfrags and reference transcripts') to combine our transcripts with annotations from five well-established databases, Refseq (ref_GRCh37.p13_top_level.gtf), Ensembl (Ensembl_Homo_sapiens.GRCh37.75.gtf), UCSC (hg19), Gencode (gencode.v19.annotation.gtf) and the lncRNA database NONCODE 4.0 (NONCODEv4u1 human_lncRNA.gtf) [44-49]; (2) unannotated transcripts were acquired based on the overlap of the combined transcripts, and BEDTools (version 2.18) was used to eliminate transcripts that had at least one exon overlapping with annotations from any of the five databases; (3) transcripts $>200$ bp were then selected $[16,50]$ and (4) novel lncRNAs were acquired based on non-coding potential by integrating the results from the four prediction tools: iseeRNA, CPAT, CPC and PLEK [51-54].

\section{Estimating relative expression and differential expression analysis}

A matrix of gene expression levels across all samples was obtained by computing the expression levels of Refseq coding genes and lncRNAs (both novel lncRNAs and the annotated lncRNAs) with Cuffquant and Cuffnorm [39]. The annotations of Refseq coding genes and annotated lncRNAs were directly downloaded from the highly reliable database: Refseq and NONCODE V4.

The $\mathrm{R}$ package Monocle was used to conduct differential expression tests between any two consecutive 
stages. Differential expression of a specific gene between any two consecutive stages was noted if the $\log _{2}$ fold change was $>1$ and the false discovery rate-adjusted $P$-value indicated statistical significance $(q$-value $<0.01)$ after the Benjamini-Hochberg correction for multiple testing $[43,55]$.

\section{Temporal specificity analysis}

\section{Normalization of expression vectors for temporal specificity calculation}

To calculate the temporal specificity scores of genes, the expression vector needed to be converted to an abundance density. First, the raw FPKM (fragments per kilobase of exons per million fragments mapped) of each gene was converted to $\log _{10}(\mathrm{FPKM}+1)$. Then this expression vector was normalized to a density vector by formula 3 , in which $V=\left(\mathrm{v}_{1}, \ldots, \mathrm{v}_{\mathrm{n}}\right)$ is the original raw FPKM abundance estimation of each gene and $V^{\prime}$ is the new normalized density vector [41].

$$
V^{\prime}=\frac{\log _{10}(V+1)}{\sum_{i=1}^{n} \log _{10}\left(v_{i}+1\right)}
$$

\section{Calculating temporal specificity score}

To calculate the temporal specificity score, we used an entropy-based measure to quantify the similarity between a gene's expression pattern and another predefined pattern that represents an extreme case in which a gene was expressed during only one stage [41]. The entropy of a discrete probability distribution was calculated by formula 4 .

$$
H(p)=-\sum_{i=1}^{n} p_{i} \log \left(p_{i}\right), \text { s.t } \quad p=\left(p_{1}, p_{2}, . ., p_{n}\right), 0 \leq p_{i} \leq 1, \sum_{i=1}^{n} p_{i}=1
$$

The temporal specificity score was defined by formula 5 , in which $J S_{\text {dist }}$ dist was the Jensen-Shannon distance (JSD) between two stage expression patterns and e was the gene expression pattern across $\mathrm{n}$ stages (formula 6). And $e^{s}$ was a predefined expression pattern that represented the extreme case in which a gene was expressed in only one stage. It was defined by formula (7).

$$
\begin{aligned}
& J S_{s p}(e \mid s)=1-J S_{\text {dist }}\left(e, e^{s}\right) \\
& J S_{\mathrm{dist}}\left(e^{1}+e^{2}\right)=\sqrt{H\left(\frac{e^{1}+e^{2}}{2}\right)-\frac{H\left(e^{1}\right)+H\left(e^{2}\right)}{2}} \\
& e^{s}=\left(e_{1}^{s}, . ., e_{n}^{s}\right), \text { s.t } \quad e_{i}^{s}=\left\{\begin{array}{ll}
1 & \text { if } i=s \\
0 & \text { otherwise }
\end{array}\right\}
\end{aligned}
$$

Finally, the temporal specificity score of a gene was defined as the maximal temporal specificity score across all $n$ stage of the genes expression pattern $e$ :

$$
J S_{s p}(e)=\operatorname{argmax}_{s} J S_{s p}(e \mid s), \quad s=1 . . n .
$$

\section{Neighboring gene correlation analysis}

Two genes were defined as neighbors if the distance between the gene bodies was $<10 \mathrm{~kb}$. Correlation between the expression pattern of an lncRNA and its neighbor coding gene was estimated by calculating the Pearson correlation coefficient $(P$-value $\leq 0.05)$ between their density-normalized expression vectors $[17,41]$. The neighbor genes could be divided into two categories: divergent (bidirectional) neighbor gene pairs and unidirectional neighbor gene pairs. The divergent neighbor gene pairs were identified as gene pairs that were arranged head-to-head on opposite strands [56].

\section{Analysis of IncRNA functions in human early- stage embryonic development}

\section{IncRNA co-expression network construction and gene module detection}

The R package WGCNA was used to construct an lncRNA co-expression network [57]. The stage-specific genes, those that were differentially expressed between any two consecutive stages, were selected to construct the network. A signed weighted correlation network was constructed by first creating a matrix of Pearson correlation coefficients between all pairs of genes across the measured samples. Second, an adjacency matrix was calculated by raising the correlation matrix to power $\beta=5$. The power of 5 was the soft threshold of the correlation matrix and made the adjacency network exhibit approximate scale-free topology $\left(R^{2}=0.9\right)$. To minimize effects of noise and spurious associations, the adjacency matrix was transformed into a Topological Overlap Matrix (TOM). Genes with highly similar coexpression relationships were grouped together by performing average linkage hierarchical clustering on the topological overlap. Dynamic Hybrid Tree Cut algorithm was used to cut the hierarchal clustering tree and define modules as branches from the tree cutting. The expression profile of each module was represented by its first principal component (module eigengene), which could explain the most variation in the module expression levels. Modules with highly correlated module eigengenes (correlation $>0.85$ ) were merged together.

\section{Identification and visualization of hub genes}

The module membership (also known as module eigengene based connectivity, $\mathrm{kME}$ ) of each gene was calculated by correlating the gene expression profile with module eigengenes with formula 9 , in which $x_{i}$ is the gene expression profile of gene $\mathrm{i}$ and $M E^{q}$ is the module eigengene of the module $q$ [57].

$$
k M E_{q}(i)=\operatorname{cor}\left(x_{i}, M E^{q}\right)
$$


Genes with the highest module membership values were referred to as intramodular hub genes (kME $\geq 0.9, P$-value $<10^{-22}$ ). Intramodular hub genes, which were centrally located inside the module, represent the expression profiles of the entire module and reflect the core functions of the module [57]. We used VisANT to visualize the gene connections (based on topological overlap) among the intramodular hub genes [58].

\section{Interaction analysis of hub genes}

LncTar, a reliable bioinformatics tool, was used to analyze the interaction between hub lncRNAs and hub coding genes in each module [59]. The variation on the standard "sliding" algorithm approach was utilized to calculate the normalized binding free energy (ndG) and identify the minimum free energy joint structure. The ndG was regard as a cutoff (ndG $\leq-0.1$ ) to determine the paired RNAs as either interacting or not. The accuracy of LncTar is over $80 \%$ confirmed by the biological experiments [59].

\section{Module preservation statistics}

To compare human and mouse lncRNA modules, we mapped human genes to the orthologous mouse gene annotations from the Mouse Genome Informatics (MGI) database [60]. The function 'overlapTableUsingKME' in the WGCNA R package was used to assess whether two modules were preserved based on a hypergeometric test that uses kME [61].

\section{Function enrichment analysis}

Gene Ontology (GO) enrichment and Kyoto Encyclopedia of Genes and Genomes (KEGG) pathway analysis of modules was carried out with the $\mathrm{R}$ packages GOstats and org.Hs.eg.db. Hypergeometric tests were applied with a $P$-value cut-off of 0.05 and minimum gene count of 5. Each module was tested for GO enrichment in terms of the Biological Process categories [62, 63].

\section{CONFLICTS OF INTEREST}

None.

\section{GRANT SUPPORT}

This work was supported by grants from the National Basic Research Project of China (2014CB964701 and 2014CB964703) and the National Natural Science Foundation of China (81471485).

\section{REFERENCES}

1. Niakan KK, Han J, Pedersen RA, Simon C, Pera RAR. Human pre-implantation embryo development. Development. 2012; 139:829-841.
2. Dean W, Lucifero D, Santos F. DNA methylation in mammalian development and disease. Birth Defects Res C Embryo Today. 2005; 75:98-111.

3. Rivera RM, Ross JW. Epigenetics in fertilization and preimplantation embryo development. Progress in Biophysics and Molecular Biology. 2013; 113:423-432.

4. Cheedipudi S, Genolet O, Dobreva G. Epigenetic inheritance of cell fates during embryonic development. Frontiers in Genetics. 2014; 5.

5. Alves Vidigal J, Ventura A. Embryonic stem cell miRNAs and their roles in development and disease. Semin Cancer Biol. 2012; 22:428-436.

6. Grote P, Herrmann BG. The long non-coding RNAFendrr links epigenetic control mechanisms to gene regulatory networks in mammalian embryogenesis. Rna Biol. 2014; 10:1579-1585.

7. Huynh KD, Lee JT. Inheritance of a pre-inactivated paternal X chromosome in early mouse embryos. Nature. 2003; 426:857-862.

8. Okamoto I, Arnaud D, Le Baccon P, Otte AP, Disteche CM, Avner P, Heard E. Evidence for de novo imprinted $\mathrm{X}$-chromosome inactivation independent of meiotic inactivation in mice. Nature. 2005; 438:369-373.

9. Chen LL, Zhao JC. Functional analysis of long noncoding RNAs in development and disease. Adv Exp Med Biol. 2014; 825:129-158.

10. Bortolin-Cavaille ML, Cavaille J. The SNORD115 (H/ MBII-52) and SNORD116 (H/MBII-85) gene clusters at the imprinted Prader-Willi locus generate canonical box C/D snoRNAs. Nucleic Acids Res. 2012; 40:6800-6807.

11. Alders M, Bliek J, Vd Lip K, Vd Bogaard R, Mannens M. Determination of KCNQ1OT1 and H19 methylation levels in BWS and SRS patients using methylation-sensitive high-resolution melting analysis. Eur J Hum Genet. 2008; 17:467-473.

12. Pauli A, Valen E, Lin MF, Garber M, Vastenhouw NL, Levin JZ, Fan L, Sandelin A, Rinn JL, Regev A, Schier AF. Systematic identification of long noncoding RNAs expressed during zebrafish embryogenesis. Genome Res. 2012; 22:577-591.

13. Caballero J, Gilbert I, Fournier E, Gagn D, Scantland S, Macaulay A, Robert C. Exploring the function of long noncoding RNA in the development of bovine early embryos. Reproduction, Fertility and Development. 2015; 27:40.

14. Nitsche A, Rose D, Fasold M, Reiche K, Stadler PF. Comparison of splice sites reveals that long noncoding RNAs are evolutionarily well conserved. RNA. 2015; 21:801-812.

15. Xue Z, Huang K, Cai C, Cai L, Jiang C, Feng Y, Liu Z, Zeng Q, Cheng L, Sun YE, Liu J, Horvath S, Fan G. Genetic programs in human and mouse early embryos revealed by single-cell RNAsequencing. Nature. 2013; 500:593-597.

16. Yan L, Yang M, Guo H, Yang L, Wu J, Li R, Liu P, Lian Y, Zheng X, Yan J, Huang J, Li M, Wu X, et al. Single-cell 
RNA-Seq profiling of human preimplantation embryos and embryonic stem cells. Nat Struct Mol Biol. 2013; 20:1131-1139.

17. Zhang K, Huang K, Luo Y, Li S. Identification and functional analysis of long non-coding RNAs in mouse cleavage stage embryonic development based on single cell transcriptome data. Bmc Genomics. 2014; 15:845.

18. Ponjavic J, Oliver PL, Lunter G, Ponting CP. Genomic and Transcriptional Co-Localization of Protein-Coding and Long Non-Coding RNA Pairs in the Developing Brain. Plos Genet. 2009; 5:e1000617.

19. Ørom UA, Derrien $\mathrm{T}$, Beringer M, Gumireddy $\mathrm{K}$, Gardini A, Bussotti G, Lai F, Zytnicki M, Notredame C, Huang Q, Guigo R, Shiekhattar R. Long Noncoding RNAs with Enhancer-like Function in Human Cells. Cell. 2010; 143:46-58.

20. Kornienko AE, Guenzl PM, Barlow DP, Pauler FM. Gene regulation by the act of long non-coding RNA transcription. Bmc Biol. 2013; 11:59.

21. Saskova A, Solc P, Baran V, Kubelka M, Schultz RM, Motlik J. Aurora kinase A controls meiosis I progression in mouse oocytes. Cell Cycle. 2008; 7:2368-2376.

22. Yoon SJ, Kim EY, Kim YS, Lee HS, Kim KH, Bae J, Lee KA. Role of Bcl2-like 10 (Bc12110) in Regulating Mouse Oocyte Maturation. Biol Reprod. 2009; 81:497-506.

23. Assou S. The human cumulus-oocyte complex geneexpression profile. Hum Reprod. 2006; 21:1705-1719.

24. Yu C, Ji S, Sha Q, Sun Q, Fan H. CRL4-DCAF1 ubiquitin E3 ligase directs protein phosphatase 2A degradation to control oocyte meiotic maturation. Nat Commun. 2015; 6:8017.

25. Huo L, Fan H, Zhong Z, Chen D, Schatten H, Sun Q. Ubiquitin-proteasome pathway modulates mouse oocyte meiotic maturation and fertilization via regulation of MAPK cascade and cyclin B1 degradation. Mech Develop. 2004; 121:1275-1287.

26. Østrup O, Andersen IS, Collas P. Chromatin-linked determinants of zygotic genome activation. Cell Mol Life Sci. 2013; 70:1425-1437.

27. Van Blerkom J. Mitochondrial function in the human oocyte and embryo and their role in developmental competence. Mitochondrion. 2011; 11:797-813.

28. Chappel S. The Role of Mitochondria from Mature Oocyte to Viable Blastocyst. Obstetrics and Gynecology International. 2013; 2013:1-10.

29. Kannan S, Rogozin IB, Koonin EV. MitoCOGs: clusters of orthologous genes from mitochondria and implications for the evolution of eukaryotes. Bmc Evol Biol. 2014; 14:237.

30. Berdasco M, Esteller M. Genetic syndromes caused by mutations in epigenetic genes. Hum Genet. 2013; 132:359-383.

31. Huo LJ, Fan HY, Zhong ZS, Chen DY, Schatten H, Sun QY. Ubiquitin-proteasome pathway modulates mouse oocyte meiotic maturation and fertilization via regulation of MAPK cascade and cyclin B1 degradation. Mech Dev. 2004; 121:1275-1287.

32. Beall S, Brenner C, Segars J. Oocyte maturation failure: a syndrome of bad eggs. Fertil Steril. 2010; 94:2507-2513.

33. Bohmdorfer G, Wierzbicki AT. Control of Chromatin Structure by Long Noncoding RNA. Trends Cell Biol. 2015; 25:623-632.

34. Maruyama A, Mimura J, Itoh K. Non-coding RNA derived from the region adjacent to the human HO-1 E2 enhancer selectively regulates HO-1 gene induction by modulating Pol II binding. Nucleic Acids Res. 2014; 42:13599-13614.

35. Prensner JR, Iyer MK, Sahu A, Asangani IA, Cao Q, Patel L, Vergara IA, Davicioni E, Erho N, Ghadessi M, Jenkins RB, Triche TJ, Malik R, et al. The long noncoding RNA SChLAP1 promotes aggressive prostate cancer and antagonizes the SWI/SNF complex. Nat Genet. 2013; 45:1392-1398.

36. Xue Z, Huang K, Cai C, Cai L, Jiang C, Feng Y, Liu Z, Zeng Q, Cheng L, Sun YE, Liu J, Horvath S, Fan G. Genetic programs in human and mouse early embryos revealed by single-cell RNAsequencing. Nature. 2013; 500:593-597.

37. Kim D, Langmead B, Salzberg SL. HISAT: a fast spliced aligner with low memory requirements. Nat Methods. 2015; 12:357-360.

38. Luo H, Sun S, Li P, Bu D, Cao H, Zhao Y. Comprehensive characterization of 10,571 mouse large intergenic noncoding RNAs from whole transcriptome sequencing. Plos One. 2013; 8:e70835.

39. Trapnell C, Williams BA, Pertea G, Mortazavi A, Kwan G, van Baren MJ, Salzberg SL, Wold BJ, Pachter L. Transcript assembly and quantification by RNA-Seq reveals unannotated transcripts and isoform switching during cell differentiation. Nat Biotechnol. 2010; 28:511-515.

40. Guttman M, Garber M, Levin JZ, Donaghey J, Robinson J, Adiconis X, Fan L, Koziol MJ, Gnirke A, Nusbaum C, Rinn JL, Lander ES, Regev A. Ab initio reconstruction of cell type-specific transcriptomes in mouse reveals the conserved multi-exonic structure of lincRNAs. Nat Biotechnol. 2010; 28:503-510.

41. Cabili MN, Trapnell C, Goff L, Koziol M, Tazon-Vega B, Regev A, Rinn JL. Integrative annotation of human large intergenic noncoding RNAs reveals global properties and specific subclasses. Gene Dev. 2011; 25:1915-1927.

42. Robin X, Turck N, Hainard A, Tiberti N, Lisacek F, Sanchez J, Müller M. pROC: an open-source package for $\mathrm{R}$ and $\mathrm{S}+$ to analyze and compare ROC curves. Bmc Bioinformatics. 2011; 12:77.

43. Sun L, Zhang Z, Bailey TL, Perkins AC, Tallack MR, Xu Z, Liu H. Prediction of novel long non-coding RNAs based on RNA-Seq data of mouse K1f1 knockout study. Bmc Bioinformatics. 2012; 13:331.

44. Wang L, Zhou D, Tu J, Wang Y, Lu Z. Exploring the stability of long intergenic non-coding RNA in K562 cells 
by comparative studies of RNA-Seq datasets. Biol Direct. 2014; 9:15.

45. Xie C, Yuan J, Li H, Li M, Zhao G, Bu D, Zhu W, Wu W, Chen R, Zhao Y. NONCODEv4: exploring the world of long non-coding RNA genes. Nucleic Acids Res. 2013; 42:D98-D103.

46. Cunningham F, Amode MR, Barrell D, Beal K, Billis K, Brent S, Carvalho-Silva D, Clapham P, Coates G, Fitzgerald S, Gil L, Giron CG, Gordon L, et al. Ensembl 2015. Nucleic Acids Res. 2015; 43:D662-D669.

47. Rosenbloom KR, Armstrong J, Barber GP, Casper J, Clawson H, Diekhans M, Dreszer TR, Fujita PA, Guruvadoo L, Haeussler M, Harte RA, Heitner S, Hickey G, et al. The UCSC Genome Browser database: 2015 update. Nucleic Acids Res. 2015; 43:D670-D681.

48. Pruitt KD, Tatusova T, Maglott DR. NCBI reference sequences (RefSeq): a curated non-redundant sequence database of genomes, transcripts and proteins. Nucleic Acids Res. 2007; 35:D61-D65.

49. Harrow J, Frankish A, Gonzalez JM, Tapanari E, Diekhans M, Kokocinski F, Aken BL, Barrell D, Zadissa A, Searle S, Barnes I, Bignell A, Boychenko V, et al. GENCODE: The reference human genome annotation for The ENCODE Project. Genome Res. 2012; 22:1760-1774.

50. Quinlan AR, Hall IM. BEDTools: a flexible suite of utilities for comparing genomic features. Bioinformatics. 2010; 26:841-842.

51. Wang L, Park HJ, Dasari S, Wang S, Kocher JP, Li W. CPAT: Coding-Potential Assessment Tool using an alignment-free logistic regression model. Nucleic Acids Res. 2013; 41:e74.

52. Kong L, Zhang Y, Ye ZQ, Liu XQ, Zhao SQ, Wei L, Gao G. CPC: assess the protein-coding potential of transcripts using sequence features and support vector machine. Nucleic Acids Res. 2007; 35:W345-W349.

53. Sun $K$, Chen $X$, Jiang $P$, Song $X$, Wang $H$, Sun $H$. iSeeRNA: identification of long intergenic non-coding
RNA transcripts from transcriptome sequencing data. BMC Genomics. 2013; 14:S7.

54. Li A, Zhang J, Zhou Z. PLEK: a tool for predicting long non-coding RNAs and messenger RNAs based on an improved k-mer scheme. Bmc Bioinformatics. 2014; 15:311.

55. Trapnell C, Cacchiarelli D, Grimsby J, Pokharel P, Li S, Morse M, Lennon NJ, Livak KJ, Mikkelsen TS, Rinn JL. The dynamics and regulators of cell fate decisions are revealed by pseudotemporal ordering of single cells. Nat Biotechnol. 2014; 32:381-386.

56. Trinklein ND. An Abundance of Bidirectional Promoters in the Human Genome. Genome Res. 2003; 14:62-66.

57. Langfelder P, Horvath S. WGCNA: an R package for weighted correlation network analysis. Bmc Bioinformatics. 2008; 9:559.

58. Hu Z, Chang YC, Wang Y, Huang CL, Liu Y, Tian F, Granger B, DeLisi C. VisANT 4.0: Integrative network platform to connect genes, drugs, diseases and therapies. Nucleic Acids Res. 2013; 41:W225-W231.

59. Li J, Ma W, Zeng P, Wang J, Geng B, Yang J, Cui Q. LncTar: a tool for predicting the RNA targets of long noncoding RNAs. Brief Bioinform. 2015; 16:806-812.

60. Eppig JT, Blake JA, Bult CJ, Kadin JA, Richardson JE. The Mouse Genome Database (MGD): comprehensive resource for genetics and genomics of the laboratory mouse. Nucleic Acids Res. 2011; 40:D881-D886.

61. Langfelder P, Luo R, Oldham MC, Horvath S. Is my network module preserved and reproducible? Plos Comput Biol. 2011; 7:e1001057.

62. Falcon S, Gentleman R. Using GOstats to test gene lists for GO term association. Bioinformatics. 2007; 23:257-258.

63. Carlson M. org.Hs.eg.db: Genome wide annotation for Human. R package version 3.2.3. 